\title{
LAS POLÍTICAS DE VEJEZ \\ EN CASTILLA-LA MANCHA (1995-2005) \\ UN POSIBLE ESCENARIO
}

\section{INTRODUCCIÓN}

El presente artículo resume la investigación social que se ha realizado en la comunidad de Castilla-La Mancha, en el marco de la Universidad regional, con el fin de conocer los posibles escenarios futuros de las políticas sociales de vejez, en la sociedad castellanomanchega. Para alcanzar este objetivo se consultó, mediante una encuesta longitudinal prospectiva y de opinión, a 160 personas seleccionadas entre representantes políticos, sindicales, de asociaciones de ancianos y de organizaciones no gubernamentales, así como entre profesionales de los servicios sociales.

En el apartado de resultados se presentan los más relevantes, estructurados en cinco áreas de estudio: pensiones, salud y asistencia sanitaria, servicios sociales, cultura y ocio, y participación.

Las conclusiones y recomendaciones que aparecen al final de este artículo recogen los fundamentos principales para una posible reorientación de las políticas de vejez en

\footnotetext{
* José María Bleda García, Doctor en Sociología y Profesor de la Universidad de Castilla-La Mancha.
} 
Castilla-La Mancha. Políticas sociales que han de fomentar los aspectos positivos de la vejez y la jubilación, a la vez que deben promover y desarrollar actuaciones para que los mayores tengan una vida más activa y decidan por sí mismos como desean vivir.

\section{MATERIAL Y MÉTODOS}

La investigación se realizó en la Universidad de Castilla-La Mancha, Facultad de Económicas, Área de Sociología, campus de Albacete, durante un periodo de dos años (1994-1995).

El procedimiento científico empleado en esta investigación ha sido el método Delphi (Delfos, oráculo de); método de previsión científica y tecnológica desarrollado en la década de los sesenta en investigaciones acerca de la predicción tecnológica de hechos futuros. Hemos seleccionado este método porque al existir anonimato entre los participantes proporciona al agrupamiento de sus respuestas al cuestionario, un destacado valor de contraste e independencia entre posiciones muy representativas del arco de valores, intereses y actitudes de los principales grupos beligerantes y creadores de opinión en el ámbito de los servicios sociales.

La técnica que se ha aplicado en esta investigación social ha sido el cuestionario; el cual se ha enviado mediante correo postal, con el fin de que el experto se autoadministrase el cuestionario y respondiese al mismo. La encuesta es longitudinal prospectiva y de opinión, siendo la mayoría de las preguntas cerradas (68) y sólo 4 abiertas. La encuesta se remitió a un grupo de expertos que se agrupaban en cuatro paneles: legisladores y políticos (35), profesionales del sector (77), asociaciones de ancianos (37) y organizaciones no gubernamentales (11).

El cuestionario elaborado pretendía, primordialmente, conocer las principales bases de sustentación para una posible reorientación de las políticas de vejez en la comunidad castellano-manchega, y sus contenidos versaban sobre el sistema de pensiones, salud y asistencia sanitaria, servicios sociales, cultura y ocio, y participación. Respecto al sistema de pensiones las preguntas iban relacionadas sobre los recursos de que disponen las personas mayores, su inquietud ante el futuro, el nivel de ingresos, y las acciones que podían llevarse a cabo para mejorar las pensiones. En cuanto a las preguntas sobre salud y asistencia sanitaria se intentaba recoger la opinión sobre las medidas que se iban a poner en marcha a través de los planes gerontológicos nacional y regional para mejorar el nivel de salud de las personas mayores. 
Un tercer grupo de aspectos eran los relacionados con las prestaciones y los servicios sociales. Se demandaba la opinión de los expertos sobre las actuaciones sociales que se iban a desarrollar en la próxima década en la comunidad castellano-manchega y que tuvieran relación con el servicio de ayuda a domicilio, los centros abiertos, la autonomía personal, las estructuras urbanas, las residencias, etc.

Otro aspecto que considerábamos necesario conocer era el relativo al acceso de las personas mayores a los bienes culturales, para lo que interrogamos a los expertos sobre una serie de acciones que podían llevarse a cabo en la Comunidad y en concreto sobre el grado de implicación de la Administración cara a facilitar que nuestros mayores elevasen su nivel cultural y formativo, el aprovechamiento de la riqueza cultural de los mayores, el turismo social, el deporte, las relaciones intergeneracionales, etc.

Las últimas preguntas iban dirigidas a conocer las tendencias y líneas de desarrollo futuro en el área de participación. Se enmarcaron, en primer lugar, en el grado de implicación de los mayores en las distintas instituciones y organizaciones de tercera edad y, en segundo lugar, en el papel de la Administración Pública en el fomento de la participación social de los mayores.

\section{RESULTADOS}

A continuación pasamos a exponer los resultados obtenidos en el desarrollo de la investigación empírica, una vez analizadas las respuestas de los panelistas colaboradores, en el estudio sobre las tendencias y líneas de desarrollo de las políticas de vejez en CastillaLa Mancha durante el periodo 1995-2005.

Los resultados se han estructurado en las cinco áreas estudiadas y reflejan el posible escenario en el que se desarrollarán las políticas de vejez a lo largo de los próximos diez años, según el consenso alcanzado entre la mayoría de los expertos consultados.

\section{Pensiones}

- Existirá solidaridad con las personas mayores que no tengan recursos económicos suficientes si hay un gobierno progresista. 
- Se protegerá a las personas de más de 80 años, que han perdido su autonomía personal, complementando su pensión y facilitándoles ayudas técnicas para mejorar su entorno y su calidad de vida, apoyando para esto a la familia, a los cuidadores y al voluntariado, y poniendo a su disposición los medios adecuados para prestar una atención más personalizada y más humanizada. Cuando ello no pueda ser, este grupo de personas debería disponer de (residencias asistidas, miniresidencias, centros de día, viviendas tuteladas...) en donde se les puedan atender sus necesidades básicas.

- Se equiparará la cuantía de la pensión mínima familiar al salario mínimo interprofesional.

- Las pensiones de viudedad se igualarán a las pensiones de jubilación.

- Se revalorizarán de una manera automática todas las pensiones de acuerdo con la evolución del IPC.

- Todos los pensionistas recibirán el mismo número de pagas anuales.

- El Estado seguirá haciéndose cargo del pago de las pensiones de los jubilados, aunque se debería realizar un mayor control para evitar el fraude en la concesión de pensiones.

- Existirá un sistema mixto en el que las pensiones públicas se complementarán con pensiones privadas.

- Los planes privados de jubilación aumentarán muy rápidamente.

En relación al análisis de las grandes tendencias de los distintos grupos de expertos con respecto a las pensiones hemos de comentar, que el grupo que se muestra más en desacuerdo con la opinión mayoritaria es el de las organizaciones no gubernamentales, pues opinan que la solidaridad con las personas mayores que carezcan de rentas económicas suficientes y no hayan cotizado o no tengan cubierto por el tiempo necesario los periodos establecidos, se obtendrá con un gobierno conservador y no con un gobierno progresista, y consideran que el Estado, en el futuro, no podrá pagar las pensiones a todos los jubilados, opinión que comparte igualmente el grupo de los profesionales. Donde no existe tampoco un consenso generalizado es en la cuestión planteada sobre si se cree que los planes privados de jubilación aumentarán muy rápidamente, pues aunque la tendencia es al acuerdo mayoritario, se da un alto porcentaje de indecisos en el grupo de las asociaciones de mayores y en el de los legisladores y políticos. 


\section{Salud y asistencia sanitaria}

- En los centros de atención primaria de salud, las personas mayores estarán atendidas mediante programas individualizados de medicina preventiva, programas de educación sanitaria, programas de medicina asistencial específicos y programas de asistencia sanitaria a domicilio.

- Los profesionales sanitarios contarán con programas de formación geriátrica y gerontológica.

- La atención sanitaria al anciano estará protocolizada.

- En el domicilio de las personas mayores se prestarán servicios médicos y de enfermería.

- Los familiares y los cuidadores de los mayores tendrán una formación en cuidados sanitarios geriátricos.

- En cada provincia de la región castellano-manchega se contará con una Unidad hospitalaria geriátrica.

- Los ancianos que residan en instituciones asistenciales dispondrán de los servicios sanitarios adecuados a sus necesidades.

Como resumen de las grandes tendencias por grupos de expertos hay que resaltar, que de las medidas que se han señalado que se pueden alcanzar en la Comunidad en el año 2005 , los grupos opinan que están de acuerdo en la gran mayoría de ellas. Siendo él que más difiere el de los profesionales, ya que está en desacuerdo con que se vayan a implantar, en los centros de salud, los programas individualizados de medicina preventiva y se vaya a dar atención integral domiciliaria a los pacientes terminales, opinión que también comparte el grupo de las organizaciones no gubernamentales. Además, el grupo de los profesionales se encuentra indeciso ante la implantación de los programas de educación sanitaria, de los programas de interconsultas entre niveles asistenciales y de la formación sobre cuidados a familiares y cuidadores. El grupo de las asociaciones de mayores sólo se encuentra dudoso en cuanto al desarrollo de la atención integral domiciliaria a pacientes terminales, servicio que el grupo de los legisladores no cree que se llegue a implantar, al igual que el de los programas de interconsultas entre los niveles de asistencia. 


\section{Servicios sociales}

- Desde los servicios sociales de la comunidad autónoma se van a realizar actuaciones en relación con la investigación sobre la vejez y el envejecimiento, la formación geriátrica y gerontológica de profesionales y la creación de un banco de datos $\mathrm{y}$ de fondos documentales sobre las personas mayores.

- Se fomentará la responsabilidad ciudadana y la convivencia intergeneracional mediante campa as de sensibilización para estimular el voluntariado, a la vez que se pretende motivar a las personas mayores para su participación en acciones de voluntariado.

- La política social desde la comunidad autónoma va a ir enmarcada en torno a las siguientes actuaciones: desarrollo y generalización de las prestaciones económicas no periódicas; mayor habitabilidad de los domicilios; reserva de un porcentaje de las viviendas de protección oficial; promoción de las viviendas tuteladas; desarrollo del programa de «acogida familiar»; generalización y cualificación del servicio de ayuda a domicilio; extensión de la red de centros abiertos; fomento de la ayuda y el apoyo a las familias que cuidan algún anciano; potenciación de las ayudas técnicas y nuevas tecnologías para facilitar la autonomía personal de los mayores discapacitados; eliminación de las barreras arquitectónicas de los edificios públicos; humanización de las estructuras urbanas; facilitar la accesibilidad a los transportes públicos; atención a la demanda de residencias termales; $y$, garantizar una plaza residencial.

- Se potenciará el mantenimiento de las personas mayores en su domicilio a través de la solidaridad familiar, el voluntariado, el desarrollo del sector privado, las ayudas a las familias, y sobre todo a través de la generalización y cualificación del servicio de ayuda a domicilio.

Atendiendo a las grandes tendencias para cada uno de los grupos de expertos, podemos observar como en el área de servicios sociales el consenso es muy alto, y las diferencias encontradas son mínimas. Las divergencias se producen, principalmente, en el grupo de las organizaciones no gubernamentales, en el que se considera que en la próxima década no van a tener importancia las actuaciones de política social en la Comunidad de CastillaLa Mancha, referidas a la humanización de las estructuras urbanas, la accesibilidad a los transportes públicos y la garantía de una plaza residencial a quien la necesite. El grupo de las asociaciones de mayores se aparta del consenso general y estima que las actuaciones 
que se realicen en relación a la humanización de las estructuras urbanas no van a tener importancia, y que el sector privado no se va a desarrollar de una manera importante, opinión, esta última, que comparte el grupo de profesionales del sector.

\section{Cultura y ocio}

En cuanto a la política en cultura y ocio, los grupos de expertos prevén que en un futuro próximo la administración regional, junto con la administración estatal, tenderá a: facilitar que nuestros mayores aumenten su nivel cultural e instructivo; potenciar que en los centros gerontológicos se incremente el nivel de educación y cultura; favorecer los desplazamientos urbanos e interurbanos; aprovechar la riqueza cultural de nuestros mayores; fomentar la práctica del turismo; facilitar que se reduzcan los precios y se donen localidades para asistir a actos culturales; promocionar la asistencia a instalaciones deportivas para su participación activa; realizar programas y campañas de sensibilización; programar periódicamente cursos y seminarios; fomentar en el profesorado y en el alumnado el interés y el conocimiento del envejecimiento; e, incorporar a los programas de formación profesional especialidades en geriatría y gerontología.

Atendiendo a la opinión de los diferentes grupos se constata nuevamente el consenso generalizado también en el área de cultura y ocio, solamente el grupo de las organizaciones no gubernamentales y el de los legisladores difieren de los demás en que se encuentran indecisos sobre si se va a fomentar o no en el profesorado y el alumnado el interés y el conocimiento del envejecimiento, asimismo el primero de estos grupos está dubitativo sobre si se va o no a promocionar la asistencia a instalaciones deportivas a los ancianos para su participación activa.

\section{Participación}

En el área de participación, la previsión es que las personas mayores participarán más activamente en las instituciones políticas y sindicales, en las organizaciones sociales, en los centros específicos de tercera edad y en los órganos de representación y asesoramiento de la administración pública. Al mismo tiempo, desde la Administración se impulsará la participación social y política de nuestros mayores, potenciando su capacidad de participación en los centros gerontológicos, generalizando la implantación de la participación democrática en dichos centros, y estableciendo órganos de representación y asesoramiento que garanticen la participación de los mayores. 
En cuanto al parecer de los distintos grupos de expertos se observa una vez más el acuerdo generalizado y únicamente se encuentran en desacuerdo en relación a la cuestión de si la Administración va a establecer órganos de representación y asesoramiento que aseguren la participación, destacando que si bien todos los grupos están mayoritariamente de acuerdo en que así va a ser, hay que señalar que el grupo de legisladores se halla indeciso, así como un alto porcentaje de las organizaciones no gubernamentales y de los profesionales.

\section{DISCUSIÓN}

Hasta aquí hemos podido contemplar como pueden ser las tendencias y líneas de desarrollo de las políticas de vejez en la próxima década en Castilla-La Mancha, con lo que creemos haber alcanzado los objetivos que nos planteamos al principio de la investigación. No obstante, considero conveniente exponer unas conclusiones y/o recomendaciones sobre las políticas de vejez en la comunidad castellano-manchega.

En primer lugar, sí bien es cierto que contamos con un marco normativo europeo, estatal y regional para desarrollar unas políticas adecuadas a la realidad de la población anciana actual, e incluso esto así es percibido por los actores implicados en ello, y que además creen que así va a suceder, sería preciso que esas bases, directrices o recomendaciones se especificaran y objetivaran más concretamente en el caso de Castilla-La Mancha. Se debería ultimar el Plan Gerontológico Regional (actualmente en discusión) en el que se considerarán los objetivos a conseguir, con qué medios y cuál sería la implicación de la Administración pública en todos sus niveles. Ahora bien, este Plan debería formar parte de las políticas sociales que emprenda la Administración dirigidas a la población en general, a todas las edades, y en los aspectos de formación, empleo, tiempo libre y de transferencias sociales.

En el aspecto económico es necesario que aumenten las cuantías de las pensiones mínimas equiparando la cuantía mínima familiar al salario mínimo interprofesional, equiparando las pensiones mínimas de viudedad con las pensiones de jubilación, revalorizando automáticamente todas las pensiones de acuerdo con la evolución del IPC y estableciendo el mismo número de pagas anuales para todos los pensionistas.

En relación a otras cuestiones sobre política de pensiones, resaltar que no existe una tendencia clara y definida sobre si el Estado podrá pagar pensiones a todos los jubilados, y el debate actual sobre ello se reproduce igualmente en Castilla-La Mancha. 
En cuanto a los servicios sanitarios sería necesario disponer, en la comunidad castellano-manchega, de una oferta de servicios que comprendan la atención preventiva y asistencial, que probablemente se tendrán en la próxima década, si tenemos en cuenta no sólo las previsiones del Plan de Salud regional, elaborado por la Consejería de Sanidad.

La atención sanitaria a domicilio dirigida a las personas mayores debería coordinarse con los servicios sociales para que se pudieran realizar actuaciones sectorizadas. Coordinación que podría, a su vez, permitir una generalización y racionalización de los servicios de ayuda a domicilio, así como la racionalización en la creación de plazas institucionales, y un aumento de la calidad en la atención a los ancianos institucionalizados.

Así mismo, el Plan Gerontológico y el Plan de Salud regionales prevén un aumento de los recursos hospitalarios y domiciliarios, postura refrendada, como veíamos anteriormente, por la mayoría de los expertos, y que si bien estamos de acuerdo con esta medida, en algunos casos lo que se debería hacer es reorientar estos recursos e intentar que resultasen más eficientes.

Por último, no hay que olvidar que un pilar básico en la atención sanitaria es la formación especializada tanto de los sanitarios como de los familiares y de los cuidadores, y para ellö desde la Administración, sería preciso poner en funcionamiento programas de formación geriátrica y gerontológica. Cuestión ésta que si bien se considera fundamental, no existe un elevado consenso, entre los consultados, a cerca de la posibilidad de que estas acciones se realicen en los años venideros.

Otras actuaciones sobre política social en la comunidad castellano-manchega que se deberían acometer son las referidas a desarrollo y generalización de las prestaciones económicas no periódicas, mayor habitabilidad de los domicilios, reserva de un porcentaje de las viviendas de protección oficial, viviendas tuteladas, desarrollo del programa de acogida familiar, generalización y cualificación del servicio de ayuda a domicilio, extensión de la red de centros abiertos, fomento de la ayuda y apoyo a las familias que cuidan a algún mayor, facilitar ayudas técnicas y nuevas tecnologías, supresión de barreras arquitectónicas, humanización de las estructuras urbanas, accesibilidad a los transportes públicos, atención de la demanda en residencias termales y garantía de una plaza residencial.

Por otra parte, el hecho de que estas actuaciones se llevaran a cabo, supondría un elevado aumento del gasto social, por lo que desde la administración regional deberían definirse de una manera más exacta y adecuada los problemas reales del colectivo de los 
mayores. Teniendo en cuenta no sólo la limitación de los recursos, sino también porque, probablemente, las demandas van a ser distintas de las actuales, y los comportamientos socioculturales serán también diferentes a los actuales, sería conveniente evaluar las necesidades de la población anciana, contemplando sus diferentes dimensiones, y con el fin de que se dispusiera de una panorámica global tanto de los individuos como de los grupos. Como consecuencia habría que racionalizar el gasto, estableciendo un sistema de prioridades y efectuando una gestión eficiente, así como la cofinanciación de ciertos servicios por parte de los.usuarios.

La solidaridad familiar, el voluntariado y el mantener a las personas mayores en su medio habitual, son aspectos que también deberían ser desarrollados, ya que son actuaciones que facilitarían el mantenimiento del anciano en su entorno, evitando lo más posible la institucionalización y favoreciendo alternativas de alojamiento.

En las nuevas formas de alojamiento (viviendas comunitarias y pisos tutelados) que se están implantando actualmente en Castilla-La Mancha, y que se van a desarrollar en un futuro próximo, se ha de intentar no trasladar el modelo residencias en su organización y funcionamiento, para lo que los mayores han de tener una mayor autonomía y participación, con el fin de evitar su «dependencia», aislamiento y desarraigo.

A la hora de aplicar estas políticas sociales de intervención comunitaria, la Administración debería tener en cuenta la heterogeneidad social, económica y personal de los ancianos; y contemplar los diferentes déficits de las personas mayores, como son la deficiente información sobre los recursos disponibles, la inadaptación a la nueva forma de vida, el gran desconocimiento de lo que hoy supone realmente la vejez, la fuerte presencia de los problemas de salud, y la aparición de un tiempo libre dominante.

Otros aspectos que la Comunidad Autónoma y la Administración Local deben tener en cuenta a la hora de establecer sus políticas de vejez son los referidos a facilitar que nuestros mayores eleven su nivel cultural y formativo. Para ello, deberían favorecer los deplazamientos urbanos e interurbanos, fomentar el turismo social y facilitar la asistencia a actos culturales; aprovechando, a su vez, la riqueza cultural de que disponen las personas mayores.

De la misma manera, se debe contemplar en el Plan Gerontológico nacional y en el regional la sensibilización de la sociedad sobre aspectos del envejecimiento y la vejez. 
Una cuestión más que está recogida en los planes gerontológicos nacional y regional, y por lo tanto susceptible de ser desarrollada, es la de la participación de los mayores en las distintas instituciones y organizaciones de la tercera edad, y la del papel de la administración pública en el fomento de la participación social de la población anciana. Las personas mayores verán, probablemente, incrementada su participación actual en las organizaciones políticas, sindicales, ciudadanas, en centros específicos de tercera edad, y en los órganos de representación y asesoramiento de la Administración pública, según el criterio del $56,07 \%$ de los expertos consultados.

Del mismo modo la Administración deberá fomentar la intervención social y política de los mayores, potenciando su capacidad de participación en los centros gerontológicos, implantando la participación democrática en dichos centros, y estableciendo en los distintos niveles de la administración órganos de representación y asesora miento que garanticen la participación real de los ancianos.

Otras actividades que la comunidad autónoma prevé desarrollar próximamente son la creación de una unidad administrativa dirigida a la investigación y docencia sobre la vejez y el envejecimiento, y el fomento de la responsabilidad de los ciudadanos y la convivencia intergeneracional.

Por último, se debe reconocer la importancia de la iniciativa privada y de las organizaciones no gubernamentales en la prestación de servicios a la población anciana, sectores estos con los que la Administración pública debería establecer una colaboración estrecha y coordinada.

En definitiva, las políticas sociales han de fomentar los aspectos positivos de la vejez y la jubilación, promoviendo y desarrollando medidas para que los mayores realicen una vida activa, con autonomía personal, a la vez que se les estimula para que se organicen y decidan por sí mismos cómo vivir. 


\section{BIBLIOGRAFíA}

- ALBER, J., GUILLEMARD, A.M. y WALKER, A., (1991): Les politiques sociales et économiques et les personnes âgées, Premier reaport annuel de l'observatoire de la CE, Comisión de la UE, Bruselas.

- ARANGUREN, J.L. (1992): La vejez como autorrealización personal y social, Ministerio de Asuntos Sociales, INSERSO, Madrid.

- AA.VV. (1985): Política social y crisis económica. Aproximación a la experiencia española, Siglo XXI, Madrid.

- AA.VV., (1991): Estado, privatización y bienestar. Un debate de la Europa actual, en: Rodríguez Cabrero (comp.), Icaria y Fuhem, Barcelona y Madrid.

- AA.VV., (1992): Política Social y Estado del Bienestar,. Ministerio de Asuntos Sociales, Madrid.

- AA.VV., (1992): Evaluación e intervención psicológica en la vejez, Martínez Roca, S.A., Barcelona.

- AA.VV., (1993): Intercambio social y desarrollo del bienestar, en: Moreno, L. (comp.),C.S.I.C., Madrid.

- BAZO, M.T., (1990): La sociedad anciana, C.I.S., Madrid.

- BAZO, M.T.: "La familia como elemento fundamental en la salud y el bienestar de las personas ancianas", Revista Española de Geriatría y Gerontología, 1, (1991): 47-52.

- CABRÉ, A. y PÉREZ, J., (1995): “Envejecimiento demográfico en España”, en SECOT (edit.). Las actividades económicas de las personas mayores, Seniors españoles para la cooperación técnica,Madrid.

- CÁRCELES, G. y MONREAL, J., (1995): "Cambio social en España y políticas para los mayores en el contexto europeo”, en: SECOT (edit.). Las actividades económicas de las personas mayores, Seniors españoles para la cooperación técnica, Madrid.

- CASTELLS, M. y ORTIZ, L.P., (1992): Análisis de las políticas de vejez en España en el contexto europeo, INSERSO, Madrid.

- C.I.S., (1990): Situación social de los viejos en España, Centro de Investigaciones Sociológicas, Madrid.

- DALKEY, N.C. et alt., (1972): La previsión a long terme par le methode delphi, Dunod, Paris.

- FERNÁNDEZ-BALLESTEROS, R., (1992): Mitos y realidades sobre la vejez y la 
salud, S.G. Editores, Barcelona, y Caja de Madrid.

- GARCÍA COTARELO, R.: "Proceso histórico del bienestar social: la consolidación del Estado del Bienestar", Revista de estudios sociales y de sociología aplicada, Documentación Social, 71, (1988):17-38.

- GUILLEMARD, A.M., (1990): Analyse comparée des Politiques de la Vieillesse en Europe, Instituto Universitario de Sociología de las Nuevas Tecnologías, Universidad Autónoma de Madrid.

- HELMER, O.: "An experimental applicattion of the delphi method to the use experts", Management Sciencie, 9, (1963).

- JUNTA DE COMUNIDADES DE CASTILla-LA MANCHA, (1994): Plan Gerontológico Regional, borrador elaborado por la Consejería de Bienestar Social, Toledo. JUNTA DE COMUNIDADES DE CASTILLA-LA MANCHA, (1995): Plan de Salud, Consejería de Sanidad y Consumo (aprobado por Consejo de Gobierno el 20-IV-95), Toledo.

- MiShaRA, R., (1984): The welfare state in crisis, Sussex: Harvester Press.

- MORAGAS, R., (1991): Gerontología social. Envejecimiento y calidad de vida, Herder, Barcelona.

- OCDE. (1990): "El envejecimiento demográfico.Consecuencias para la política social”, en: El futuro de la protección social y el envejecimiento de la población, Ministerio de Trabajo y Seguridad Social, Madrid.

- PÉREZ ORTIZ, L.P., (1995): “Evolución de las políticas públicas para la vejez”, en: SECOT(edit), Las actividades económicas de las personas mayores, Seniors españoles para la cooperación técnica, Madrid.

- PICÓ, J., (1990): Teorías sobre el Estado del Bienestar, Siglo XXI, Madrid.

- PILL, J.: "The delphi method: substance, context, a critique an annotated bibliography", Socio Economic Planing Sciences, 5, (1971).

- RODRÍGUEZ CABRERO, G., (1992): “Fundamentos teóricos de la política social”, en: Moreno, L. (ed.), Política Social y Estado del Bienestar, Ministerio de Asuntos Sociales, Madrid.

- SÁNCHEZ VERA, P.: “ Bases y fundamentos para una aproximación sociológica a la vejez”, Papers, 40, (1992):100-117.

- SÁNCHEZ VERA, P., (ed.) (1993): Sociedad y población anciana, Universidad de Murcia, Murcia. 